Pure Mathematical Sciences, Vol. 3, 2014, no. 4, 153 - 156

HIKARI Ltd, www.m-hikari.com

http://dx.doi.org/10.12988/pms.2014.4716

\title{
Superderivation of the Hyperbolic Subalgebra for $\mathrm{pl}(\mathbf{m}, \mathbf{n}, \mathbf{R})$
}

\author{
Hailing $\mathrm{Li}^{1}$, Haijian $\mathrm{Cui}^{2}$ and Ying Wang ${ }^{3}$ \\ ${ }^{1}$ School of Science, Shandong University of Technology, Zibo, 255049, China \\ ${ }^{2}$ Nanchang Institute of Science and Technology, Nanchang, 330108, China \\ ${ }^{3}$ School of Science, Dalian University of Technology, Dalian, 116023, China \\ Copyright (c) 2014 Hailing Li, Haijian Cui and Ying Wang. This is an open access article \\ distributed under the Creative Commons Attribution License, which permits unrestricted \\ use, distribution, and reproduction in any medium, provided the original work is properly \\ cited.
}

\begin{abstract}
Let $R$ be an arbitrary commutative ring with $1,1 / 2 \in R, \operatorname{pl}(m, n, R)$ the general linear Lie superalgebra over $R$. The aim of this paper is to give an explicit description of the superderivation algebras of the hyperbolic subalgebra of $\mathrm{pl}(m, n, R)$.
\end{abstract}

Mathematics Subject Classification: 17B30; 17B40

Keywords: hyperbolic subalgebra; superderivation; commutative ring

\section{Introduction}

If $\operatorname{deg}(x)$ occurs in some expression, then $x$ is assumed to be a homogeneous element denoted by $\operatorname{hg}(x)$ and $\operatorname{deg}(x)$ is the degree of $x$ about $Z_{2}$-gradation.

Let $R$ be a commutative ring with $1,1 / 2 \in R$ and $L$ a Lie superalgebra over $R$. Recall that an $R$-linear mapping $\phi: L \rightarrow L$ is called a $Z_{2}$-homogeneous Lie superderivation of degree $\theta$ if

$$
\phi([a, b])=[\phi(a), b]+(-1)^{\operatorname{deg}(\mathrm{a}) \theta}[a, \phi(b)] \quad \forall a, b \in L_{\overline{0}} \cup L_{\overline{1}} .
$$

A Lie superderivation is the sum of a Lie superderivation of degree $\overline{0}$ and a Lie superderivation of degree $\overline{1}$. 
In recently years, many significant researches have been done in derivations of general linear Lie algebra and its subalgebras (see [1-2]). In this paper, we will describe the hyperbolic subalgebras of $\operatorname{pl}(m, n, R)$ and give an explicit description on the superderivations for the hyperbolic subalgebras of $\operatorname{pl}(m, n, R)$ to $\operatorname{pl}(m, n, R)$. We obtain that any Lie superderivation $\phi$ for the hyperbolic subalgebras of $\operatorname{pl}(m, n, R)$ to $\operatorname{pl}(m, n, R)$ can be uniquely written as the sum of an inner superderivation and an induced superderivation.

\section{The hyperbolic subalgebras of $\mathbf{p l}(m, n, R)$}

Put $l=m+n$. Throughout this paper, $\operatorname{pl}(m, n, R)$ denotes the $R$-algebra of all $l \times l$ matrices over a commutative ring $R$. It is obvious that $\left\{E_{i j} \mid 1 \leq i, j \leq l\right\}$ is a basis of $\mathrm{pl}(m, n, R)$, where $E_{i j}$ is the matrix whose sole nonzero entry is 1 in the $(i, j)$ position. The bracket operation

$$
\left[E_{i j}, E_{k t}\right]=\delta_{j k} E_{i t}-(-1)^{(\tau(i)+\tau(j))(\tau(k)+\tau(t))} \delta_{i t} E_{k j},
$$

where $\tau(i)=\overline{0}$ for $i \in\{1,2, \ldots, m\}, \tau(i)=\overline{1}$ for $i \in\{m+1, m+2, \ldots, l\}$.

Define $\operatorname{deg}\left(E_{i j}\right)=\tau(i)+\tau(j)$ on $\operatorname{pl}(m, n, R)$ a structure of Lie superalgebra over $R$. We denote by $\mathrm{D}(m, n, R)$ the Lie superalgebra consisting of all diagonal matrices.

Definition 2.1 Let $\Phi=\left\{A_{i, j} \in I(R) \mid 1 \leq i, j \leq l\right\}$ be a subset of $I(R)$ consisting of $l^{2}$ ideals of $R$. We call $\Phi$ a flag of ideals of $R$ if and only if for any $i, j, k$ :

(1) $A_{i, i}=R, i=1,2, \cdots, l$;

(2) $A_{i, k} A_{k, j} \subseteq A_{i, j}$.

Example 2.2 If $i \neq j$, let $A_{i, i}=R$ for $i=1,2, \cdots, l$. Then $\Phi=\left\{A_{i, j} \in\right.$ $I(R) \mid 1 \leq i, j \leq l\}$ is a flag of ideals of $R$.

Example 2.3 If all $A_{i, j}, 1 \leq i, j \leq l$ are taken to be $R$, then $\Phi=\left\{A_{i, j} \in\right.$ $I(R) \mid 1 \leq i, j \leq l\}$ is a flag of ideals of $R$.

Example 2.4 Set $A_{i, i}=R$ for $i=1,2, \cdots, l$. Let $A_{1,2}, A_{2,3}, \cdots, A_{l-1, l}$, $A_{2,1}, A_{3,2}, \cdots, A_{l, l-1}$ be any ideals of $R$ respectively, and set

$$
\begin{array}{ll}
A_{i, j}=\prod_{k=1}^{j-i} A_{i+k-1} A_{i+k}, & 1 \leq i<j \leq l, \\
A_{i, j}=\prod_{k=1}^{j-i} A_{i-k+1} A_{i-k}, & 1 \leq j<i \leq l .
\end{array}
$$

Then $\Phi=\left\{A_{i, j} \in I(R) \mid 1 \leq i, j \leq l\right\}$ is a flag of ideals of $R$. 
Definition 2.5 Any subalgebra of $p l(m, n, R)$ containing $D(m, n, R)$ is called a hyperbolic subalgebra.

Lemma 2.6 $L$ is a hyperbolic subalgebra of $p l(m, n, R)$ if and only if there exists a flag $\Phi=\left\{A_{i, j} \in I(R) \mid 1 \leq i, j \leq l\right\}$ of ideals of $R$ such that $L=L_{\Phi}=$ $\sum_{1 \leq i, j \leq l} A_{i, j} E_{i, j}$.

\section{Main results}

Let $L_{\Phi}=\sum_{1 \leq i, j \leq l} A_{i, j} E_{i, j}$ be a fixed hyperbolic subalgebra of $\operatorname{pl}(m, n, R)$ with $\Phi=\left\{A_{i, j} \in I(R) \mid 1 \leq i, j \leq l\right\}$ a flag of ideals of $R$. In order to show any superderivation of $L_{\Phi}$ to $\operatorname{pl}(m, n, R)$, in this section we construct certain superderivations of $L_{\Phi}$ to $\operatorname{pl}(m, n, R)$ as follows.

(I) Inner superderivation

Let $x \in \operatorname{pl}(m, n, R)$, then ad : $L_{\Phi} \rightarrow \operatorname{pl}(m, n, R), y \mapsto[x, y]$ is a superderivation of $L_{\Phi}$ to $\operatorname{pl}(m, n, R)$, called the inner superderivation of $L_{\Phi}$ to $\operatorname{pl}(m, n, R)$ induced by $x$.

\section{(II) Induced superderivation}

Let $\Sigma=\left\{\sigma_{i, j} \in \operatorname{Hom}_{R}\left(A_{i, j}, R\right) \mid 1 \leq i, j \leq l\right\}$ be a set consisting of $l^{2}$ homomorphisms of $R$-modules. We call $\Sigma$ suitable for induced superderivations if for any $i, j, k$ :

(1) $\sigma_{i, i}(r)=\lambda_{i} r$, where $\lambda_{i}, r \in R$,

(2) $\sigma_{i, j}\left(a_{i, k} a_{k, j}\right)=\sigma_{i, k}\left(a_{i, k}\right) a_{k, j}+a_{i, k} \sigma_{k, j}\left(a_{k, j}\right)$. where $a_{i, k} \in A_{i, k}, a_{k, j} \in A_{k, j}$.

Using the homomorphism $\Sigma=\left\{\sigma_{i, j} \in \operatorname{Hom}_{R}\left(A_{i, j}, R\right) \mid 1 \leq i, j \leq l\right\}$ which is suitable for induced superderivations, we define $\Phi_{\Sigma}: L_{\Phi} \rightarrow \operatorname{pl}(m, n, R)$ by sending any $\sum_{1 \leq i, j \leq l} a_{i, j} E_{i, j}$ to $\sum_{1 \leq i, j \leq l} \sigma_{i, j}\left(a_{i, j}\right) E_{i, j}$.

Lemma 3.1 $\Phi_{\Sigma}$ is a Lie superderivation of $L_{\Phi}$ to $p l(m, n, R)$.

If $l=1$, there is nothing to do, so we only consider the case $l \geq 2$. Let $W$ be the subset of $\operatorname{pl}(m, n, R)$ consisting of all the matrices whose diagonal entries are all 0 . In the following, we describe any Lie superderivation of the hyperbolic subalgebra $L_{\Phi}$ to $\mathrm{pl}(m, n, R)$.

Theorem 3.2 Let $R$ be a commutative ring with $1,1 / 2 \in R, L_{\Phi}=\sum_{1 \leq i, j \leq l} A_{i, j} E_{i, j}$ a fixed hyperbolic subalgebra of $p l(m, n, R)$ containing $D(m, n, R)$ with $\Phi=$ $\left\{A_{i, j} \in I(R) \mid 1 \leq i, j \leq l\right\}$ a flag of ideals of $R$. Then each superderivation of $L_{\Phi}$ to $p l(m, n, R)$ may be uniquely written as the sum of an induced superderivation and an inner superderivation induced by an element in $W$. 


\section{References}

[1] H. Wang, Q. Li, Lie triple derivations of the Lie algebra of strictly upper triangular matrices over a commutative ring, Linear Algebra and its Applications, 430(2009) 66-77.

[2] H. Li, Y. Wang, Genearlized Lie triple derivations, Linear and Multilinear Algebra, 59(2011) 237-247.

Received: July 1, 2014 